\title{
ON THE GENERALIZED WEIGHTED INTEGRAL INEQUALITY FOR DOUBLE INTEGRALS
}

\author{
BY
}

\author{
MEHMET ZEKI SARIKAYA
}

\begin{abstract}
In this paper, we obtain weighted Montgomery's identities for function of two variables and apply them to give new generalization weighted integral inequality for double integrals involving functions of two independent variables by using fairly elementary analysis.
\end{abstract}

Mathematics Subject Classification 2010: 26D07, 26D15.

Key words: Ostrowski's inequality, Montgomery's identities, double integrals.

\section{Introduction}

In 1938, the classical integral inequality established by Ostrowski [8] as follows:

Theorem 1. Let $f:[a, b] \rightarrow \mathbb{R}$ be a differentiable mapping on $(a, b)$ whose derivative $f^{\prime}:(a, b) \rightarrow \mathbb{R}$ is bounded on $(a, b)$, i.e.,

$$
\left\|f^{\prime}\right\|_{\infty}=\sup _{t \in(a, b)}\left|f^{\prime}(t)\right|<\infty .
$$

Then, the inequality holds:

$$
\left|f(x)-\frac{1}{b-a} \int_{a}^{b} f(t) d t\right| \leq\left[\frac{1}{4}+\frac{\left(x-\frac{a+b}{2}\right)^{2}}{(b-a)^{2}}\right](b-a)\left\|f^{\prime}\right\|_{\infty},
$$

for all $x \in[a, b]$. The constant $\frac{1}{4}$ is the best possible. 
Inequality (1.1) has wide applications in numerical analysis and in the theory of some special means; estimating error bounds for some special means, some mid-point, trapezoid and Simpson rules and quadrature rules, etc. Hence inequality (1.1) has attracted considerable attention and interest from mathematicans and researchers. Due to this, over the years, the interested reader is also refered to ([1]-[7],[9]-[20]) for integral inequalities in several independent variables. In addition, the current approach of obtaining the bounds, for a particular quadrature rule, have depended on the use of Peano kernel. The general approach in the past has involved the assumption of bounded derivatives of degree greater than one.

If $f:[a, b] \rightarrow \mathbb{R}$ is differentiable on $[a, b]$ with the first derivative $f^{\prime}$ integrable on $[a, b]$, then Montgomery identity holds:

$$
f(x)=\frac{1}{b-a} \int_{a}^{b} f(t) d t+\int_{a}^{b} P(x, t) f^{\prime}(t) d t
$$

where $P(x, t)$ is the Peano kernel defined by

$$
P(x, t):= \begin{cases}\frac{t-a}{b-a}, & a \leq t<x \\ \frac{t-b}{b-a}, & x \leq t \leq b .\end{cases}
$$

Suppose now that $w:[a, b] \rightarrow[0, \infty)$ is some probability density function, i.e. is a positive integrable function satisfying $\int_{a}^{b} w(t) d t=1$, and $W(t)=\int_{a}^{t} w(x) d x$ for $t \in[a, b], W(t)=0$ for $t<a$ and $W(t)=1$ for $t>b$. The following identity is a generalization of Montgomery's identity,

$$
f(x)=\frac{1}{b-a} \int_{a}^{b} w(t) f(t) d t+\int_{a}^{b} P_{w}(x, t) f^{\prime}(t) d t,
$$

where $P_{w}(x, t)$ is the weighted Peano kernel defined by

$$
P_{w}(x, t):= \begin{cases}W(t), & a \leq t<x \\ W(t)-1, & x \leq t \leq b\end{cases}
$$

In [3] and [5], the authors obtain two identities which generalize (1.2) for functions of two variables. In fact, for $f:[a, b] \times[c, d] \rightarrow \mathbb{R}$ such that the partial derivative $\frac{\partial f(t, s)}{\partial t}, \frac{\partial f(t, s)}{\partial s}$, and $\frac{\partial f^{2}(t, s)}{\partial t \partial s}$ all exist and are continuous on 
$[a, b] \times[c, d]$ and for all $(x, y) \in[a, b] \times[c, d]$, they obtain:

$$
\begin{aligned}
& (d-c)(b-a) f(x, y) \\
& =-\int_{a}^{b} \int_{c}^{d} f(t, s) d s d t+(d-c) \int_{a}^{b} f(t, y) d t \\
& +(b-a) \int_{c}^{d} f(x, s) d s+\int_{a}^{b} \int_{c}^{d} p(x, t) p(y, s) \frac{\partial f^{2}(t, s)}{\partial t \partial s} d s d t
\end{aligned}
$$

and

$$
\begin{aligned}
& (d-c)(b-a) f(x, y) \\
& =\int_{a}^{b} \int_{c}^{d} f(t, s) d s d t+\int_{a}^{b} \int_{c}^{d} p(x, t) \frac{\partial f(t, s)}{\partial t} d s d t \\
& +\int_{a}^{b} \int_{c}^{d} q(y, s) \frac{\partial f(t, s)}{\partial s} d s d t+\int_{a}^{b} \int_{c}^{d} p(x, t) p(y, s) \frac{\partial f^{2}(t, s)}{\partial t \partial s} d s d t
\end{aligned}
$$

where

$$
p(x, t)=\left\{\begin{array}{ll}
t-a, & a \leq t<x \\
t-b, & x \leq t \leq b
\end{array} \text { and } q(y, s)= \begin{cases}s-c, & c \leq s<y \\
s-d, & y \leq s \leq d\end{cases}\right.
$$

In a recent paper [19], by using the weight function $w:[a, b] \rightarrow[0, \infty)$, is integrable, nonnegative and

$$
m(a, b)=\int_{a}^{b} w(t) d t<\infty
$$

Sarikaya and Ogunmez proved a generalization of Montgomery's identity for double integrals

Lemma 1. Let $f:[a, b] \times[c, d] \rightarrow \mathbb{R}$ be an absolutely continuous function such that the partial derivative of order 2 exists for all $(t, s) \in[a, b] \times[c, d]$. Then, we have:

$$
\begin{aligned}
f(x, y) & =\frac{1}{m(a, b)} \int_{a}^{b} w(t) f(t, y) d t+\frac{1}{m(c, d)} \int_{c}^{d} w(s) f(x, s) d s \\
& -\frac{1}{m(a, b) m(c, d)}\left[\int_{a}^{b} \int_{c}^{d} w(t) w(s) f(t, s) d s d t\right. \\
& \left.-\int_{a}^{b} \int_{c}^{d} p_{w}(x, t) q_{w}(y, s) \frac{\partial^{2} f(t, s)}{\partial t \partial s} d s d t\right],
\end{aligned}
$$


where

$$
\begin{gathered}
p_{w}(x, t)=\left\{\begin{array}{l}
p_{1}(a, t)=\int_{a}^{t} w(u) d u, \quad a \leq t<x \\
p_{2}(b, t)=\int_{b}^{t} w(u) d u, \quad x \leq t \leq b
\end{array}\right. \text { and } \\
q_{w}(y, s)=\left\{\begin{array}{l}
q_{1}(c, s)=\int_{c}^{s} w(u) d u, \quad c \leq s<y \\
q_{2}(d, s)=\int_{d}^{s} w(u) d u, \quad y \leq s \leq d .
\end{array}\right.
\end{gathered}
$$

The main aim of this paper is to establish weighted Montgomery's identities for function of two variables and apply them to give new generalization weighted integral inequality for double integrals involving functions of two independent variables by using fairly elementary analysis.

\section{Main result}

To prove our theorems, we need the following two lemmas:

Lemma 2. Let $f:[a, b] \times[c, d] \rightarrow \mathbb{R}$ have continuous partial derivatives $\frac{\partial f(t, s)}{\partial t}, \frac{\partial f(t, s)}{\partial s}$, and $\frac{\partial f^{2}(t, s)}{\partial t \partial s}$ on $[a, b] \times[c, d]$. Then, for all $(x, y) \in[a, b] \times[c, d]$, we have

$$
\begin{aligned}
f(x, y) & =\frac{1}{m(a, b) m(c, d)}\left[\int_{a}^{b} \int_{c}^{d} p_{w}(x, t) q_{w}(y, s) \frac{\partial f^{2}(t, s)}{\partial t \partial s} d s d t\right. \\
(2.1) & +\int_{a}^{b} \int_{c}^{d} p_{w}(x, t) w(s) \frac{\partial f(t, s)}{\partial t} d s d t \\
& \left.+\int_{a}^{b} \int_{c}^{d} q_{w}(y, s) w(t) \frac{\partial f(t, s)}{\partial s} d s d t+\int_{a}^{b} \int_{c}^{d} w(t) w(s) f(t, s) d s d t\right]
\end{aligned}
$$

where $p_{w}(x, t)$ and $q_{w}(y, s)$ are defined by (1.6) and (1.7), respectively.

Proof. By definitions of $p_{w}(x, t)$ and integrating by parts, we can state:

$$
f(x)=\frac{1}{m(a, b)} \int_{a}^{b} p_{w}(x, t) f^{\prime}(t) d t+\frac{1}{m(a, b)} \int_{a}^{b} w(t) f(t) d t,
$$

for all $x \in[a, b]$. Applying the identity (2.2) for the partial derivative $\frac{\partial f(t, y)}{\partial t}$ gives

$$
f(x, y)=\frac{1}{m(a, b)} \int_{a}^{b} p_{w}(x, t) \frac{\partial f(t, y)}{\partial t} d t+\frac{1}{m(a, b)} \int_{a}^{b} w(t) f(t, y) d t
$$


for all $(x, y) \in[a, b] \times[c, d]$. Again, formula (2.2) applied for the partial derivative $\frac{\partial f(t, s)}{\partial s}$ provides

$$
f(t, y)=\frac{1}{m(c, d)} \int_{c}^{d} q_{w}(y, s) \frac{\partial f(t, s)}{\partial s} d s+\frac{1}{m(c, d)} \int_{c}^{d} w(s) f(t, s) d s,
$$

for all $(t, y) \in[a, b] \times[c, d]$. Similarly, for partial the derivative of (2.4) according to $t$, we get

$$
\frac{\partial f(t, y)}{\partial t}=\frac{1}{m(c, d)} \int_{c}^{d} q_{w}(y, s) \frac{\partial f^{2}(t, s)}{\partial t \partial s} d s+\frac{1}{m(c, d)} \int_{c}^{d} w(s) \frac{\partial f(t, s)}{\partial t} d s
$$

for all $(t, y) \in[a, b] \times[c, d]$. Subsituting (2.4) and (2.5) into identity (2.3) and rewriting, we easily deduce:

$$
\begin{aligned}
f(x, y) & =\frac{1}{m(a, b) m(c, d)}\left[\int_{a}^{b} \int_{c}^{d} p_{w}(x, t) q_{w}(y, s) \frac{\partial f^{2}(t, s)}{\partial t \partial s} d s d t\right. \\
2.6) & +\int_{a}^{b} \int_{c}^{d} p_{w}(x, t) w(s) \frac{\partial f(t, s)}{\partial t} d s d t \\
& \left.+\int_{a}^{b} \int_{c}^{d} q_{w}(y, s) w(t) \frac{\partial f(t, s)}{\partial s} d s d t+\int_{a}^{b} \int_{c}^{d} w(t) w(s) f(t, s) d s d t\right]
\end{aligned}
$$

which completes the proof.

Remark 1. If we choose $w()=$.1 in Lemma 2, we get the identity (1.4).

Lemma 3. Under the assumptions of Lemma 2, we have

$$
\begin{aligned}
& \frac{f(a, c)+f(a, d)+f(b, c)+f(b, d)}{4}=\frac{1}{m(a, b) m(c, d)} . \\
& \cdot\left[\frac{1}{4} \int_{a}^{b} \int_{c}^{d}\left[p_{w}(a, t)+p_{w}(b, t)\right]\left[q_{w}(c, s)+q_{w}(d, s)\right] \frac{\partial f^{2}(t, s)}{\partial t \partial s} d s d t\right. \\
& +\frac{1}{2} \int_{a}^{b} \int_{c}^{d}\left[p_{w}(a, t)+p_{w}(b, t)\right] w(s) \frac{\partial f(t, s)}{\partial t} d s d t \\
& +\frac{1}{2} \int_{a}^{b} \int_{c}^{d}\left[q_{w}(c, s)+q_{w}(d, s)\right] w(t) \frac{\partial f(t, s)}{\partial s} d s d t \\
& \left.+\int_{a}^{b} \int_{c}^{d} w(t) w(s) f(t, s) d s d t\right] .
\end{aligned}
$$


Proof. By substituting $(x, y)=(a, c),(a, d),(b, c),(b, d)$ in $(2.1)$ respectively and summing the resulting identities and after some simplification, we get the desired identity (2.7).

Our main results are given in the following theorems:

Theorem 2. Let $f:[a, b] \times[c, d] \rightarrow \mathbb{R}$ have continuous partial derivatives $\frac{\partial f(t, s)}{\partial t}, \frac{\partial f(t, s)}{\partial s}$, and $\frac{\partial f^{2}(t, s)}{\partial t \partial s}$ on $[a, b] \times[c, d]$ and

$$
\begin{aligned}
& M_{1}=\left\|\frac{\partial f(t, s)}{\partial t}\right\|_{\infty}=\sup _{a<t<b}\left|\frac{\partial f(t, s)}{\partial t}\right|<\infty, \\
& M_{2}=\left\|\frac{\partial f(t, s)}{\partial s}\right\|_{\infty}=\sup _{c<s<d}\left|\frac{\partial f(t, s)}{\partial s}\right|<\infty, \\
& M_{3}=\left\|\frac{\partial^{2} f(t, s)}{\partial t \partial s}\right\|_{\infty}=\sup _{(t, s) \in(a, b) \times(c, d)}\left|\frac{\partial^{2} f(t, s)}{\partial t \partial s}\right|<\infty .
\end{aligned}
$$

Then, for all $(x, y) \in[a, b] \times[c, d]$, we have

$$
\begin{aligned}
& \left|f(x, y)-\frac{1}{m(a, b) m(c, d)} \int_{a}^{b} \int_{c}^{d} w(t) w(s) f(t, s) d s d t\right| \\
& \leq \frac{M_{1}}{m(a, b)}\left(\int_{a}^{x}(x-u) w(u) d u+\int_{x}^{b}(u-x) w(u) d u\right) \\
& +\frac{M_{2}}{m(c, d)}\left(\int_{c}^{y}(y-u) w(u) d u+\int_{y}^{d}(u-y) w(u) d u\right) \\
& +\frac{M_{3}}{m(a, b) m(c, d)}\left(\int_{a}^{x}(x-u) w(u) d u+\int_{x}^{b}(u-x) w(u) d u\right) \\
& \times\left(\int_{c}^{y}(y-u) w(u) d u+\int_{y}^{d}(u-y) w(u) d u\right) .
\end{aligned}
$$

Proof. From Lemma 2, using the properties of modulus, and from the hypotheses, we observe that

$$
\begin{aligned}
& \left|f(x, y)-\frac{1}{m(a, b) m(c, d)} \int_{a}^{b} \int_{c}^{d} w(t) w(s) f(t, s) d s d t\right| \\
& \leq \frac{1}{m(a, b) m(c, d)}\left\{\int_{a}^{b} \int_{c}^{d}\left|p_{w}(x, t)\right|\left|p_{w}(y, s)\right|\left|\frac{\partial f^{2}(t, s)}{\partial t \partial s}\right| d s d t\right. \\
& +\int_{a}^{b} \int_{c}^{d}\left|p_{w}(x, t)\right|\left|\frac{\partial f(t, s)}{\partial t}\right| w(s) d s d t
\end{aligned}
$$




$$
\begin{aligned}
& \left.+\int_{a}^{b} \int_{c}^{d}\left|p_{w}(y, s)\right|\left|\frac{\partial f(t, s)}{\partial s}\right| w(t) d s d t\right\} \\
& \leq \frac{1}{m(a, b) m(c, d)}\left\{M_{1} \int_{a}^{b} \int_{c}^{d}\left|p_{w}(x, t)\right| w(s) d s d t\right. \\
& \left.+M_{2} \int_{a}^{b} \int_{c}^{d}\left|p_{w}(y, s)\right| w(t) d s d t+M_{3} \int_{a}^{b} \int_{c}^{d}\left|p_{w}(x, t)\right|\left|p_{w}(y, s)\right| d s d t\right\} .
\end{aligned}
$$

First we calculate the right-hand side of the corresponding inequality (2.9), we have $\left(\int_{a}^{b}\left|p_{w}(x, t)\right| d t\right)\left(\int_{c}^{d} w(s) d s\right)=m(c, d) \int_{a}^{b}\left|p_{w}(x, t)\right| d t$, using the change of order of integration

$$
\begin{aligned}
\int_{a}^{b}\left|p_{w}(x, t)\right| d t & =\int_{a}^{x}\left|p_{1}(a, t)\right| d t+\int_{x}^{b}\left|p_{2}(b, t)\right| d t \\
& =\int_{a}^{x} \int_{a}^{t} w(u) d u d t+\int_{x}^{b} \int_{t}^{b} w(u) d u d t \\
& =\int_{a}^{x} w(u) \int_{u}^{x} d t d u+\int_{x}^{b} w(u) \int_{x}^{u} d t d u \\
& =\int_{a}^{x}(x-u) w(u) d u+\int_{x}^{b}(u-x) w(u) d u
\end{aligned}
$$

by similar computations we have

$$
\left(\int_{a}^{b} w(t) d t\right)\left(\int_{c}^{d}\left|q_{w}(y, s)\right| d s\right)=m(a, b) \int_{c}^{d}\left|q_{w}(y, s)\right| d s,
$$

and

$$
\begin{aligned}
\int_{c}^{d}\left|q_{w}(y, s)\right| d s & =\int_{c}^{y}\left|p_{1}(c, s)\right| d s+\int_{y}^{d}\left|p_{2}(d, s)\right| d s \\
& =\int_{c}^{y}(y-u) w(u) d u+\int_{y}^{d}(u-y) w(u) d u
\end{aligned}
$$

On the other hand, for the last integral of right hand side of (2.9), using the results of (2.10) and (2.11)

$$
\int_{a}^{b} \int_{c}^{d}\left|p_{w}(x, t)\right|\left|q_{w}(y, s)\right| d s d t=\left(\int_{a}^{b}\left|p_{w}(x, t)\right| d t\right)\left(\int_{c}^{d}\left|q_{w}(y, s)\right| d s\right)
$$




$$
\begin{aligned}
& =\left(\int_{a}^{x}(x-u) w(u) d u+\int_{x}^{b}(u-x) w(u) d u\right) \\
& \times\left(\int_{c}^{y}(y-u) w(u) d u+\int_{y}^{d}(u-y) w(u) d u\right) .
\end{aligned}
$$

Thus, using (2.10), (2.11) and (2.12) in (2.9), we obtain the inequality (2.8) and the proof is completed.

Corollary 1. Under the assumptions of Theorem 2, we have

$$
\begin{aligned}
& \left|f(x, y)-\frac{1}{(b-a)(d-c)} \int_{a}^{b} \int_{c}^{d} f(t, s) d s d t\right| \\
& \leq\left[\frac{1}{4}+\frac{\left(x-\frac{a+b}{2}\right)^{2}}{(b-a)^{2}}\right] M_{1}(b-a)+\left[\frac{1}{4}+\frac{\left(y-\frac{c+d}{2}\right)^{2}}{(b-a)^{2}}\right] M_{2}(d-c) \\
& +\left[\frac{1}{4}+\frac{\left(x-\frac{a+b}{2}\right)^{2}}{(b-a)^{2}}\right]\left[\frac{1}{4}+\frac{\left(y-\frac{c+d}{2}\right)^{2}}{(b-a)^{2}}\right] M_{3}(b-a)(d-c) .
\end{aligned}
$$

Proof. If we choose $w(u)=1$ in Theorem 2 , then we get the inequality (2.13)

If we take $x=\frac{a+b}{2}$ and $y=\frac{c+d}{2}$ in Theorem 2, we can give the following corollary.

Corollary 2. Under the assumptions of Theorem 2, we have

$$
\begin{aligned}
& \left|f\left(\frac{a+b}{2}, \frac{c+d}{2}\right)-\frac{1}{m(a, b) m(c, d)} \int_{a}^{b} \int_{c}^{d} w(t) w(s) f(t, s) d s d t\right| \\
& \leq \frac{M_{1}}{m(a, b)}\left(\int_{a}^{\frac{a+b}{2}}\left(\frac{a+b}{2}-u\right) w(u) d u+\int_{\frac{a+b}{2}}^{b}\left(u-\frac{a+b}{2}\right) w(u) d u\right) \\
& +\frac{M_{2}}{m(c, d)}\left(\int_{c}^{\frac{c+d}{2}}\left(\frac{c+d}{2}-u\right) w(u) d u+\int_{\frac{c+d}{2}}^{d}\left(u-\frac{c+d}{2}\right) w(u) d u\right) \\
& +\frac{M_{3}}{m(a, b) m(c, d)}\left(\int_{a}^{\frac{a+b}{2}}\left(\frac{a+b}{2}-u\right) w(u) d u+\int_{\frac{a+b}{2}}^{b}\left(u-\frac{a+b}{2}\right) w(u) d u\right) \\
& \times\left(\int_{c}^{\frac{c+d}{2}}\left(\frac{c+d}{2}-u\right) w(u) d u+\int_{\frac{c+d}{2}}^{d}\left(u-\frac{c+d}{2}\right) w(u) d u\right) .
\end{aligned}
$$


Remark 2. If we choose $w(u)=1$ in Corollary 2, we get

$$
\begin{aligned}
& \left|f\left(\frac{a+b}{2}, \frac{c+d}{2}\right)-\frac{1}{(b-a)(d-c)} \int_{a}^{b} \int_{c}^{d} f(t, s) d s d t\right| \\
& \leq \frac{M_{1}(b-a)}{4}+\frac{M_{2}(d-c)}{4}+\frac{M_{3}(b-a)(d-c)}{16} .
\end{aligned}
$$

Theorem 3. Under the assumptions of Theorem 2, we have

$$
\begin{aligned}
& \mid \frac{f(a, c)+f(a, d)+f(b, c)+f(b, d)}{4} \\
& -\frac{1}{m(a, b) m(c, d)} \int_{a}^{b} \int_{c}^{d} w(t) w(s) f(t, s) d s d t \mid \\
& \leq \frac{M_{1}(b-a)}{2}+\frac{M_{2}(d-c)}{2}+\frac{M_{3}(b-a)(d-c)}{4} .
\end{aligned}
$$

Proof. From Lemma 3, we have

$$
\begin{aligned}
& \mid \frac{f(a, c)+f(a, d)+f(b, c)+f(b, d)}{4} \\
& -\frac{1}{m(a, b) m(c, d)} \int_{a}^{b} \int_{c}^{d} w(t) w(s) f(t, s) d s d t \mid \leq \frac{1}{m(a, b) m(c, d)} . \\
& \cdot\left[\frac{M_{3}}{4} \int_{a}^{b} \int_{c}^{d}\left[\left|p_{w}(a, t)\right|+\left|p_{w}(b, t)\right|\right]\left[\left|q_{w}(c, s)\right|+\left|q_{w}(d, s)\right|\right] d s d t\right. \\
& +\frac{M_{1}}{2} \int_{a}^{b} \int_{c}^{d}\left[\left|p_{w}(a, t)\right|+\left|p_{w}(b, t)\right|\right] w(s) d s d t \\
& \left.+\frac{M_{2}}{2} \int_{a}^{b} \int_{c}^{d}\left[\left|q_{w}(c, s)\right|+\left|q_{w}(d, s)\right|\right] w(t) d s d t\right] .
\end{aligned}
$$

By simple computations in proof of Theorem 2, we obtain inequality (2.14).

Corollary 3. Under the assumptions of Theorem 3, we have

$$
\begin{aligned}
& \mid \frac{f(a, c)+f(a, d)+f(b, c)+f(b, d)}{4} \\
& -\frac{1}{(b-a)(d-c)} \int_{a}^{b} \int_{c}^{d} f(t, s) d s d t \mid \\
& \leq \frac{M_{1}(b-a)}{2}+\frac{M_{2}(d-c)}{2}+\frac{M_{3}(b-a)(d-c)}{4} .
\end{aligned}
$$


Proof. If we choose $w()=$.1 in Theorem 3, then we get the desired inequality (2.15).

\section{REFERENCES}

1. Ahmad, F.; Barnett, N.S.; Dragomir, S.S. - New weighted Ostrowski and Čebyšev type inequalities, Nonlinear Anal., 71 (2009), 1408-1412.

2. Ahmad, F.; RafiQ, A.; Mir, N.A. - Weighted Ostrowski-Grüss type inequality for differentiable mappings, Glob. J. Pure Appl. Math., 2 (2006), 147-154.

3. BARnetT, N.S.; Dragomir, S.S. - An Ostrowski type inequality for double integrals and applications for cubature formulae, Soochow J. Math., 27 (2001), 1-10.

4. Barnett, N.S.; Dragomir, S.S.; Pearce, C.E.M. - A quasi-trapezoid inequality for double integrals, ANZIAM J., 44 (2003), 355-364.

5. Dragomir, S.S.; Cerone, P.; Barnett, N.S.; Roumeliotis, J. - An inequality of the Ostrowski type for double integrals and applications for cubature formulae, Tamsui Oxf. J. Math. Sci., 16 (2000), 1-16.

6. Dragomir, S.S.; Barnett, N.S.; Cerone, P. - An n-dimensional version of Ostrowski's inequality for mappings of the Hölder type, Kyungpook Math. J., 40 (2000), $65-75$.

7. Hussain, S.; Latif, M.A.; Alomari, M. - Generalized double-integral Ostrowski type inequalities on time scales, Appl. Math. Lett., 24 (2011), 1461-1467.

8. Ostrowski, A. - Über die Absolutabweichung einer differentiierbaren Funktion von ihrem Integralmittelwert, Comment. Math. Helv., 10 (1937), 226-227.

9. Pachpatte, B.G. - On an inequality of Ostrowski type in three independent variables, J. Math. Anal. Appl., 249 (2000), 583-591.

10. Pachpatte, B.G.- On a new Ostrowski type inequality in two independent variables, Tamkang J. Math., 32 (2001), 45-49.

11. Pachpatte, B.G. - A new Ostrowski type inequality for double integrals, Soochow J. Math., 32 (2006), 317-322.

12. Pecarić, J.; Vukelić, A. - Montgomery's identities for function of two variables, J. Math. Anal. Appl., 332 (2007), 617-630.

13. QAyYum, A. - A weighted Ostrowski-Grüss type inequality and applications, Proceeding of the World Cong. on Engineering, 2 (2009), 1-9.

14. RAfiQ, A.; Ahmad, F. - Another weighted Ostrowski-Grss type inequality for twice differentiable mappings, Kragujevac J. Math., 31 (2008), 43-51.

15. ÖZKan, U.M.; YildiRIM, H. - Ostrowski type inequality for double integrals on time scales, Acta Appl. Math., 110 (2010), 283-288. 
16. ÖZKAn, U.M.; YILDIRIM, H. - Grüss type inequalities for double integrals on time scales, Comput. Math. Appl., 57 (2009), 436-444.

17. SARIKAYA, M.Z. - On the Ostrowski type integral inequality, Acta Math. Univ. Comenian. (N.S.), 79 (2010), 129-134.

18. SARIKAyA, M.Z. - On the Ostrowski type integral inequality for double integrals, Demonstratio Math., 45 (2012), 533-540.

19. Sarikaya, M.Z.; Ogunmez, H. - On the weighted Ostrowski-type integral inequality for double integrals, Arab. J. Sci. Eng., 36 (2011), 1153-1160.

20. Ujević, N. - Some double integral inequalities and applications, Acta Math. Univ. Comenian. (N.S.), 71 (2002), 189-199.

Received: 18.IV.2012

Revised: 16.VII.2012

Accepted: 17.VII.2012
Department of Mathematics, Faculty of Science and Arts, Düzce University, Konuralp Campus, Düzce, TURKEY sarikayamz@gmail.com 